\title{
Impacts of artificial reservoirs on floristic diversity and plant functional traits in dry forests after 15 years
}

\author{
Lopes, SF. ${ }^{*}$, Vale, VS. ${ }^{b}$, Prado Júnior, JA. ${ }^{b}$ and Schiavini, I. ${ }^{b}$ \\ aPrograma de Pós-Graduação em Ecologia e Conservação, Departamento de Biologia, \\ Universidade Estadual da Paraíba - UEPB, Rua Baraúnas, 352, CEP 58109-753, Campina Grande, PB, Brazil

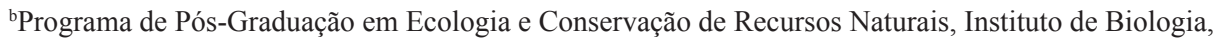 \\ Universidade Federal de Uberlandia - UFU, Rua Ceará, Umuarama, CEP 38400-902, Uberlândia, MG, Brazil \\ *e-mail: defarialopes@gmail.com
}

Received: September 13, 2013 - Accepted: March 26, 2014 - Distributed: August 31, 2015

(With 1 figure)

\begin{abstract}
Dams are of paramount importance to a wide variety of human services and many of their environmental problems are known; however, there are few studies in the world addressing the impacts on the native vegetation previously distant from water bodies which became close to the lakeshore created by a dam. Thus, this paper aims to analyze the responses of a dry forest to a dam after 15 years. For this, 20 random samples of 40 trees were made, 10 close to the lakeshore and 10 distant from it, by applying the central square point method. Close to the dam, we found higher values regarding basal area, number of trees, number of evergreen trees, and zoochoric syndrome, but there were lower values of Shannon's diversity index. Therefore, the impacts of the dam after 15 years caused several changes to the tree community. The greater basal area close to the dam suggests that water deficit during the dry season was decreased and plants have thicker trunks. On the other hand, this sector had much more zoochoric syndrome and a larger number of evergreen trees than plots which are distant from water, suggesting changes with regard to the community's ecological functions. Furthermore, structural floristic data shows that the sector close to the dam is less similar to other deciduous forests within the same geographical region than the sector distant from water, thus providing evidence of the impacts of dams on the tree community.
\end{abstract}

Keywords: phytosociology, dam impacts, dispersal, deciduousness.

\section{Impactos de um reservatório artificial sobre a diversidade florística e traços funcionais de espécies arbóreas de uma Floresta Estacional Decidual após 15 anos do represamento}

\begin{abstract}
Resumo
Barragens são de suma importância para uma ampla variedade de serviços humanos e muitos de seus problemas ambientais já são conhecidos. No entanto, existem poucos estudos no mundo abordando os impactos sobre a vegetação nativa anteriormente distante de corpos d'água, que após a barragem se aproximou do lago criado. Assim, este trabalho tem como objetivo analisar as respostas de uma floresta seca as margens da represa formada por uma barragem após 15 anos. Para isso, 20 amostras aleatórias de 40 árvores foram feitas, sendo 10 perto da margem do lago e 10 distante dele, através da aplicação do método de ponto central. Perto da barragem, encontramos valores mais elevados em relação à área basal, densidade, número de árvores perenifólias e zoocórica, mas houve menores valores de índice de diversidade de Shannon. Portanto, os impactos da barragem depois de 15 anos causou várias mudanças na comunidade de árvores. A maior área basal perto da barragem sugere que o déficit hídrico durante a estação seca foi diminuído, o que aumenta o crescimento em área basal das espécies. Por outro lado, este setor apresentou maior número de indivíduos zoocóricos e número maior de árvores perenifólias do que as parcelas que estão distantes da água, sugerindo mudanças no que diz respeito às funções ecológicas da comunidade. Além disso, os dados florísticos estruturais mostram que o setor perto da barragem é menos similar a outras florestas estacionais deciduais dentro de uma mesma região geográfica que o setor distante da água, proporcionando, assim, evidências dos impactos das barragens sobre a comunidade arbórea.
\end{abstract}

Palavras-chave: fitossociologia, impactos de barragens, síndrome de dispersão, deciduidade. 


\section{Introduction}

Historically, dams have facilitated human life; at first, with regard to farming, transportation, and domestic services and, currently, they are built to generate hydroelectric power (Evans et al., 2009). This kind of power is regarded as a clean and cheap alternative, accounting for $16 \%$ of the worldwide electricity generation in 2005 (Evans et al., 2009). Nowadays, at least 45,000 dams $>15 \mathrm{~m}$ high obstruct $60 \%$ of the fresh water flowing into the oceans (Nilsson et al., 2005).

In fact, dams are of paramount importance to a wide variety of human services. However, despite the "clean and cheap" idea attributed to them, many of their environmental problems are known (Nilsson and Berggren, 2000). Due to storage of water, natural habitat becomes fragmented (Nilsson et al., 2005), killing many elements of the flora. The decomposition of dead plants releases organic matter and depletes oxygen in water, and it also emits greenhouse gases (Giles, 2006). Notwithstanding, the impacts of a dam are not limited to the flooded area. The native vegetation previously distant from water bodies which became close to the lakeshore created by a dam represents long-term consequences, because this new "riparian vegetation" is completely distinct from an original riparian environment with regard to species and characteristics.

For instance, original riparian vegetation has species adapted to water saturation and species adapted to patches with water deficit (Bertrand et al., 2012). These environments are associated to many ecological functions, such as terrestrial-aquatic linkage of habitats, and they provide animals, such as birds and seed-dispersing mammals, with resources (Gundersen et al., 2010) and refuge (Palmer and Bennett, 2006), thus playing a key role in diversity maintenance.

However, the vegetation which became close to the lakeshore created by a dam is often located on hillsides (Truffer et al., 2003), without species associated to water saturation, and, then, with different species and distinct traits when compared to a typical riparian vegetation (Acker et al., 2003). Steep slope terrains reduce water infiltration into the soil, making it dry; species from these environments are adapted to reduce water loss, for instance, by losing leaves during the dry season or having fruits and seeds with low water content (Sidle et al., 2006). Thus, the impacts of a dam are not limited to the consequences of closeness to a water body to species adapted to water deficit. Will the "new riparian vegetation" play the same ecological role as that played by a typical riparian vegetation?

Changes to vegetation are critical because plants are primary producers and the basal component in most ecosystems (Loreau et al., 2001). However, most studies focus on dam impacts on grass, herbs, and shrubs and they are often conducted in temperate environments with low species diversity (Nilsson et al., 1997; Jansson et al., 2000; Nilsson and Svedmark, 2002; Mallik and Richardson, 2009), although most dams are built in tropical systems with high species diversity, with a predominance of trees (Guo et al., 2007).
Thus, this paper evaluates the upstream effects of the dam on a dry forest in the Southeast Brazilian region. This kind of forest is associated to mountainous or, at least, steep terrain, thus, it is an excellent object for studying changes to other forests undergoing a similar impact. This kind of forest have a marked dry season, therefore, many plant species lose their leaves in winter as a protective action against an excess of evapotranspiration (Murphy and Lugo, 1986) and diaspores with wing-like structures facilitate their seed dispersal (Vale et al., 2010). Both traits, often observed in dry environments, are unusual in humid environments, due to high air moisture, plant foliage hampers wind dispersal, and the excess volume of water is constant throughout the year. It is difficult to predict what happens when this kind of vegetation becomes close to a water body, such as a dam, as this represents a total change to the relationship of water to flora.

This paper aims to analyze the responses of a dry forest to the Miranda Hydroelectric Power Plant, in the Southeast Brazilian region, after 15 years. We evaluated two sectors of this forest; one is far from and the other is close to the reservoir margin, considering the environmental changes due to the dam. The main objectives of this paper were: i) identifying differences on the structure and parameters of flora, such as basal area, number of trees, richness and diversity in both sectors; and ii) investigating differences on the functional traits having leaf deciduousness and dispersal mechanism as a basis. We assumed the hypothesis that the impacts of the dam after 15 years caused several changes to dry forest structure, then, the sector close to the reservoir margin show more differences when compared to the sector distant to from it.

\section{Methods}

The study was carried out in a dry forest $\left(18^{\circ} 54^{\prime} 03^{\prime \prime} \mathrm{S}\right.$ and $48^{\circ} 01 ' 25^{\prime \prime} \mathrm{W}$ ) located at the margins of the Miranda Hydroelectric Power Plant (MHPP), in the town of Indianópolis, Minas Gerais, Brazil. The forest fragment has 35.4 ha and it is within the Araguari river basin. The MHPP was the second dam built in the Araguari river; it was completed in 1997, flooding a very large area. From then on, the study area undergoes the influence of water on the environment, totalizing 15 years until the time this study was conducted, in 2012. The tree community was evaluated in two sectors: A, $200 \mathrm{~m}$ far from the reservoir margin; and $\mathrm{B}$, close to the reservoir margin. At the reservoir margin, the sample points were established, since it included, the tree nearest to the dam margin.

Dry forests occur in seasonal climate, with a long dry period (from 2 to 6 months). Average annual temperature is between 23 and $25^{\circ} \mathrm{C}$ and annual rainfall ranges from 1,160 to $1,460 \mathrm{~mm}$ (Alves and Rosa, 2008). It has species exhibiting functional phenology and reproductive responses adapted to dry seasonal climate, i.e. very different from species found in tropical rainforests (Miles et al., 2006). Besides, the study area is located on a rocky and shallow soil and on a steeply declined terrain. These characteristics favor water drainage and hamper soil water storage. Besides, 
dry forests are regarded as endangered environments (Miles et al., 2006); nowadays, they are often impacted by dams, because they are associated to mountains and, thus, hydroelectric power generation.

Sampling was conducted by means of the central square point method (Cottam and Curtis, 1956). According to this method, each point means 4 sampled trees, those closest to the central square point. Thus, it was possible to calculate the phytosociological parameters (density, frequency, basal area, and importance value) for the different forest sectors (A and B). We randomly selected 10 points which served as the beginning for sampling in each forest sector. Starting from each point we sampled 10 central square points (totaling 40 trees) that were considered one sample. Then we repeated this proceeding 10 times in parallel to the dams margin, totalizing 10 samples in each sector (A - $200 \mathrm{~m}$ far from the reservoir margin; and B, close to the reservoir margin). All trees with a diameter at breast height $(\mathrm{DBH}) \geq 5 \mathrm{~cm}$ were sampled. The trees were identified at the species level and the distance from the central square point was measured. The botanic material was taken to the Laboratory of Plant Ecology of Uberlândia Federal University, and the taxonomic arrangement was based on the phylogenetic position of the APG III system (Chase and Reveal, 2009).

According to their dispersal mechanism, having fruit morphology as a basis (van der Pijl, 1982), the species were classified as zoochoric (dispersal by animals), anemochoric (dispersal by wind), and autochoric (dispersal by gravity). The species were also classified, according to the fall of leaves during the dry season, as evergreen (do not lose all leaves) or deciduous (lose all leaves), based on field observation and the literature (Lopes et al., 2012).

Phytosociological parameters were calculated for each species, using the software Fitopac 1.6 (Shepherd, 2004). We compared floristic parameters (species richness, total density, total basal area, and Shannon's diversity) to functional traits (dispersal mechanism and leaf deciduousness) by means of Student's t-test and Mann Whitney's test (non-parametric data) (Magurran, 2004).

To evaluate the $\beta$ diversity in the study area, we selected other eight studies addressing dry forests (Table 1). These forests do not suffer influence of any water course. Thus, we conducted floristic comparisons between the two forest sectors (A and B) and those from other studies, in order to simulate a floristic dissimilarity test, calculated by means of the Jaccard index and Morisita-Horn similarity index (Magurran, 2004). Through the values obtained from these indexes, we performed two clusters analysis using the method of grouping the Unweighted Pair Group Method with Arithmetic Mean (UPGMA), calculated by the software Fitopac 1.6 (Shepherd, 2004).

\section{Results}

We sampled 64 species in sector A (far from the reservoir margin) and 54 species in sector B (close to the reservoir margin). Shannon's diversity in sector A (3.40) was significantly higher than that in the sector B (3.20) (Table 2); otherwise, the basal area and the number of individuals were significantly lower in sector A $\left(23.32 \mathrm{~m}^{2} \cdot \mathrm{ha}^{-1}\right.$ and 1,542 ind.ha $\left.{ }^{-1}\right)$ than in sector B (34.43 $\mathrm{m}^{2} \cdot \mathrm{ha}^{-1}$ and 2,672 ind.ha- ${ }^{-1}$ ) (Table 2).

Regarding the functional traits, we found significant differences both in the dispersal mechanism and leaf deciduousness. Sector A showed a significantly higher anemochory ( $41 \%)$ than sector B (23\%) and lower zoochory (46\% in A and $62 \%$ in B). As for the leaf deciduousness, sector A showed to be significantly more deciduous (58\%) than B (39\%) and, at the same time, it has less evergreen trees $(42 \%$ in $A$ and $61 \%$ in $B)$.

In sector $\mathrm{A}$, the five most representative families were Anacardiaceae (23\%), Fabaceae (19\%), Rhamnaceae (8\%), Malvaceae (7\%), and Sapindaceae (5\%), representing $62 \%$ of individuals and $33 \%$ of total species (Table 3 ). In sector $\mathrm{B}$, the five most representative families were Primulaceae (22\%), Fabaceae (18\%), Anacardiaceae (14\%), Malvaceae (8\%), and Piperaceae (7\%), representing $68 \%$ of individuals and $40 \%$ of total species (Table 3 ). Caricaceae, Euphorbiaceae, Monimiaceae, Nyctaginaceae, and Rutaceae were found only in sector A. There were no families exclusive to sector B (Table 3).

Out of the 78 species sampled in both sectors, 24 species (31\%) were exclusive to sector A and 14 (18\%) were

Table 1. Structure parameters of the eight areas compared to the two sectors of this study

\begin{tabular}{cccccccc}
\hline Areas & Municipality & F & S & NI & BA & H' & References \\
\hline A & Uberlândia & 29 & 64 & 1542 & 23.32 & 3.39 & Presente estudo (TL) \\
B & Uberlândia & 24 & 54 & 2672 & 34.42 & 3.20 & Presente estudo (TP) \\
1 & Alpinópolis & 24 & 50 & 1094 & 13.49 & 2.97 & Fagundes et. al. 2007 \\
2 & Araguari & 27 & 59 & 1082 & N/Inf. & 2.84 & Souza et al 2007 \\
3 & Montes Claros & 26 & 61 & 3293 & N/Inf. & 3.30 & Santos et al., 2007 \\
4 & Passos & 21 & 39 & 550 & 18.06 & 2.97 & Fagundes et al., 2007 \\
5 & Perdizes & 46 & 114 & 1771 & 24.14 & 3.74 & Werneck et al., 2000 \\
6 & Santa Vitória & 30 & 60 & 1225 & N/Inf. & 2.89 & Carvalho et al., 1999 \\
7 & Uberlândia & 32 & 64 & 1695 & 16.25 & 2.76 & Siqueira et al., 2009 \\
8 & Uberlândia & 22 & 46 & 937 & 14.02 & 2.59 & Siqueira et al., 2009 \\
\hline
\end{tabular}

$\mathrm{F}=$ number of families; $\mathrm{S}=$ richness; $\mathrm{NI}=$ number of individuals $\left(\right.$ ind $\left._{\mathrm{h}} \mathrm{ha}^{-1}\right) ; \mathrm{BA}=$ basal area $\left(\mathrm{m}^{2} \cdot \mathrm{ha}^{-1}\right) ; \mathrm{H}^{\prime}=$ Shannon index.

$\mathrm{N} /$ Inf. $=$ No information . 
Table 2. Comparison of floristic parameters and functional traits of the tree community* at two sectors of a dry forest in the Southeast Brazilian region.

\begin{tabular}{ccccc}
\hline Parameters & Sector A** & Sector B*** & T & P \\
\hline Richness (S) & 64 & 54 & 1.03 & 0.32 \\
Shannon's diversity (H') & 3.39 & 3.20 & 2.06 & $<0.05$ \\
Basal area $\left(\mathrm{m}^{2}\right.$. ha $^{-1}$ ) & 23.32 & 34.43 & 2.53 & $<0.05$ \\
Individuals (ind.ha $^{-1}$ ) & 1,542 & 2,672 & 6.82 & $<0.05$ \\
Anemochory (\%) & 41 & 23 & 4.18 & $<0.05$ \\
Autochory (\%) & 13 & 15 & 0.26 & 0.79 \\
Zoochory (\%) & 46 & 62 & 2.84 & $<0.05$ \\
Deciduousness (\%) & 58 & 39 & 2.66 & $<0.05$ \\
Evergreen trees (\%) & 42 & 61 & 2.65 & $<0.05$ \\
\hline
\end{tabular}

$\mathrm{T}=$ value of Student's t-test; $\mathrm{P}=$ probability value of Student's t-test. $* \mathrm{DBH} \geq 5 \mathrm{~cm}$. **Distant from the reservoir margin. $* * *$ Close to the reservoir margin.

Table 3. Tree community* sampled in two sectors, $\mathrm{A}^{* *}$ and $\mathrm{B}^{* * *}$, of a dry forest in the Southeast Brazilian region, alphabetically arranged according to botanical families.

\begin{tabular}{|c|c|c|c|c|c|c|c|c|c|c|}
\hline \multirow{2}{*}{ Family/species } & \multicolumn{2}{|c|}{ RD } & \multicolumn{2}{|c|}{ RF } & \multicolumn{2}{|c|}{ RBA } & \multicolumn{2}{|c|}{ IV $(\%)$} & \multirow{2}{*}{ DS } & \multirow{2}{*}{ Dec } \\
\hline & $\mathbf{A}$ & B & $\mathbf{A}$ & B & $\mathbf{A}$ & B & $\mathbf{A}$ & B & & \\
\hline \multicolumn{11}{|l|}{ Anacardiaceae } \\
\hline Astronium fraxinifolium Schott ex Spreng. & 1.75 & 0.50 & 2.16 & 0.61 & 1.10 & 0.20 & 5.01 & 1.31 & Ane & $\mathrm{D}$ \\
\hline Lithraea molleoides (Vell.) Engl. & 1.50 & 4.25 & 1.85 & 4.86 & 1.41 & 2.29 & 4.76 & 11.40 & Zoo & $\mathrm{E}$ \\
\hline Myracrodruon urundeuva Allemão & 18.75 & 9.50 & 16.67 & 10.94 & 17.86 & 16.86 & 53.28 & 37.30 & Ane & $\mathrm{D}$ \\
\hline Tapirira guianensis Aubl. & 0.75 & - & 0.93 & - & 2.08 & - & 3.76 & - & Zoo & $\mathrm{E}$ \\
\hline \multicolumn{11}{|l|}{ Annonaceae } \\
\hline Rollinia sylvatica (A. St.-Hil.) Mart. & 0.25 & 0.50 & 0.31 & 0.61 & 0.04 & 0.49 & 0.60 & 1.60 & Zoo & $\mathrm{E}$ \\
\hline \multicolumn{11}{|l|}{ Apocynaceae } \\
\hline $\begin{array}{l}\text { Aspidosperma cuspa (Kunth) S. F. Blake ex } \\
\text { Pittier }\end{array}$ & 0.75 & - & 0.93 & - & 0.77 & - & 2.45 & - & Ane & $\mathrm{D}$ \\
\hline Aspidosperma discolor A. DC. & - & 0.25 & - & 0.30 & - & 0.03 & - & 0.59 & Ane & $\mathrm{D}$ \\
\hline Aspidosperma parvifolium A. DC. & 0.50 & - & 0.62 & - & 1.70 & - & 2.82 & - & Ane & $\mathrm{D}$ \\
\hline \multicolumn{11}{|l|}{ Araliaceae } \\
\hline $\begin{array}{l}\text { Dendropanax cuneatus (DC.) Decne. \& } \\
\text { Planch }\end{array}$ & - & 0.25 & - & 0.30 & - & 0.05 & - & 0.60 & Zoo & $\mathrm{E}$ \\
\hline Sciadodendron excelsum Griseb. & 0.25 & 0.25 & 0.31 & 0.30 & 0.33 & 1.39 & 0.89 & 1.95 & Zoo & $\mathrm{D}$ \\
\hline \multicolumn{11}{|l|}{ Arecaceae } \\
\hline $\begin{array}{l}\text { Acrocomia aculeata (Jacq.) Lodd. ex Mart. } \\
\text { Bignoniaceae }\end{array}$ & \multicolumn{9}{|c|}{ Bignoniaceae } & $\mathrm{E}$ \\
\hline $\begin{array}{l}\text { Handroanthus serratifolius (Vahl) S. O. } \\
\text { Grose }\end{array}$ & 0.50 & - & 0.62 & - & 0.27 & - & 1.39 & - & Ane & $\mathrm{D}$ \\
\hline Tabebuia roseoalba (Ridl.) Sandwith & 0.25 & 1.50 & 0.31 & 1.82 & 0.51 & 0.56 & 1.06 & 3.89 & Ane & $\mathrm{D}$ \\
\hline \multicolumn{11}{|l|}{ Burseraceae } \\
\hline Protium heptaphyllum (Aubl.) Marchand & 1.00 & 1.00 & 0.93 & 1.22 & 3.61 & 0.40 & 5.53 & 2.61 & Zoo & $\mathrm{E}$ \\
\hline \multicolumn{11}{|l|}{ Cannabaceae } \\
\hline Celtis iguanaea (Jacq.) Sarg. & 1.00 & - & 1.23 & - & 0.79 & - & 3.03 & - & Zoo & $\mathrm{D}$ \\
\hline Trema micrantha (L.) Blume & 1.00 & 0.50 & 1.23 & 0.61 & 0.32 & 0.08 & 2.56 & 1.19 & Zoo & $\mathrm{E}$ \\
\hline \multicolumn{11}{|l|}{ Caricaceae } \\
\hline Jacaratia spinosa (Aubl.) A. DC. & 0.25 & - & 0.31 & - & 0.27 & - & 0.83 & - & Zoo & $\mathrm{E}$ \\
\hline \multicolumn{11}{|l|}{ Combretaceae } \\
\hline Terminalia glabrescens Mart & 1.50 & 0.75 & 1.85 & 0.91 & 0.77 & 0.46 & 4.12 & 2.12 & Ane & $\mathrm{D}$ \\
\hline Terminalia phaeocarpa Eichler & 2.25 & 0.50 & 2.78 & 0.61 & 2.88 & 0.70 & 7.91 & 1.81 & Ane & $\mathrm{D}$ \\
\hline
\end{tabular}


Table 3. Continued...

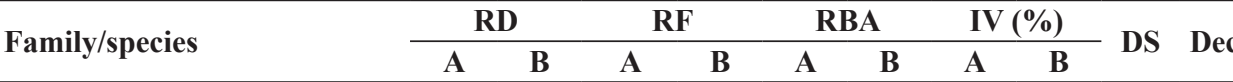

\begin{tabular}{|c|c|c|c|c|c|c|c|c|c|c|}
\hline \multicolumn{11}{|l|}{ Euphorbiaceae } \\
\hline Pera glabrata (Schott) Poepp. ex Baill. & 0.25 & - & 0.31 & - & 0.10 & - & 0.66 & - & Auto & $\mathrm{E}$ \\
\hline \multicolumn{11}{|l|}{ Fabaceae } \\
\hline Acacia polyphylla DC. & 0.25 & 1.25 & 0.31 & 1.22 & 0.46 & 0.30 & 1.02 & 2.77 & Auto & $\mathrm{D}$ \\
\hline Anadenanthera colubrina (Vell.) Brenan & 2.00 & 4.75 & 2.16 & 4.26 & 8.08 & 8.25 & 12.25 & 17.25 & Auto & $\mathrm{D}$ \\
\hline Deguelia costata (Benth.) & 9.00 & 2.75 & 5.56 & 2.13 & 3.00 & 0.65 & 17.55 & 5.53 & Ane & $\mathrm{E}$ \\
\hline $\begin{array}{l}\text { Enterolobium contortisiliquum (Vell.) } \\
\text { Morong }\end{array}$ & - & 0.75 & - & 0.91 & - & 3.89 & - & 5.55 & Auto & $\mathrm{D}$ \\
\hline Inga sessilis (Vell.) Mart. & 0.75 & 3.25 & 0.93 & 3.34 & 0.55 & 3.09 & 2.23 & 9.69 & Zoo & $\mathrm{E}$ \\
\hline Inga vera Willd. & 1.00 & 0.50 & 1.23 & 0.61 & 0.56 & 0.81 & 2.80 & 1.92 & Zoo & $\mathrm{E}$ \\
\hline $\begin{array}{l}\text { Lonchocarpus cultratus (Vell.) Az.-Tozzi \& } \\
\text { H. C. Lima }\end{array}$ & 1.75 & 2.00 & 1.85 & 2.13 & 5.37 & 3.35 & 8.97 & 7.48 & Auto & $\mathrm{D}$ \\
\hline Machaerium hirtum (Vell). Stellfeld & - & 0.50 & - & 0.61 & - & 2.79 & - & 3.90 & Ane & $\mathrm{D}$ \\
\hline Machaerium stipitatum (DC.) Vogel & 0.25 & 0.50 & 0.31 & 0.61 & 0.12 & 1.85 & 0.68 & 2.96 & Ane & $\mathrm{D}$ \\
\hline Piptadenia gonoacantha (Mart.) J. F. Macbr. & 2.50 & 1.25 & 3.09 & 1.52 & 2.41 & 1.93 & 8.00 & 4.70 & Auto & $\mathrm{D}$ \\
\hline Platypodium elegans Vogel & - & 0.25 & - & 0.30 & - & 0.14 & - & 0.69 & Ane & $\mathrm{D}$ \\
\hline Sweetia fruticosa Spreng & - & 0.25 & - & 0.30 & - & 0.15 & - & 0.70 & Ane & $\mathrm{D}$ \\
\hline Zollernia ilicifolia (Brongn.) Vogel & 1.00 & - & 1.23 & - & 0.28 & - & 2.51 & - & Auto & $\mathrm{E}$ \\
\hline \multicolumn{11}{|l|}{ Lauraceae } \\
\hline Endlicheria paniculata (Spreng.) J. F. Macbr. & 0.50 & - & 0.62 & - & 0.66 & - & 1.78 & - & Zoo & $\mathrm{E}$ \\
\hline Nectandra cissiflora Nees & 0.50 & 0.75 & 0.62 & 0.91 & 2.28 & 0.90 & 3.40 & 2.56 & Zoo & $\mathrm{E}$ \\
\hline Ocotea corymbosa (Meisn.) Mez & 0.25 & - & 0.31 & - & 0.10 & - & 0.65 & - & Zoo & $\mathrm{E}$ \\
\hline Ocotea spixiana (Nees) Mez & - & 0.50 & - & 0.61 & - & 0.16 & - & 1.27 & Zoo & $\mathrm{E}$ \\
\hline \multicolumn{11}{|l|}{ Lecythidaceae } \\
\hline Cariniana estrellensis (Raddi) Kuntze & 3.25 & 1.25 & 2.78 & 1.52 & 11.65 & 1.92 & 17.68 & 4.69 & Ane & $\mathrm{D}$ \\
\hline \multicolumn{11}{|l|}{ Malvaceae } \\
\hline Ceiba speciosa (A. St.-Hil.) Ravenna & 0.25 & - & 0.31 & - & 1.34 & - & 1.90 & - & Ane & $\mathrm{D}$ \\
\hline Guazuma ulmifolia Lam. & 6.25 & 5.25 & 6.48 & 6.38 & 5.07 & 2.12 & 17.80 & 13.75 & Auto & $\mathrm{E}$ \\
\hline Luehea divaricata Mart. & 0.25 & 2.50 & 0.31 & 2.43 & 0.21 & 0.87 & 0.77 & 5.80 & Ane & $\mathrm{D}$ \\
\hline Luehea grandiflora Mart. \& Zucc. & - & 0.25 & - & 0.30 & - & 0.06 & - & 0.62 & Ane & $\mathrm{D}$ \\
\hline Sterculia striata A. St.-Hill. \& Naudin & 0.25 & - & 0.31 & - & 0.38 & - & 0.94 & - & Zoo & $\mathrm{D}$ \\
\hline \multicolumn{11}{|l|}{ Meliaceae } \\
\hline Cedrela fissilis Vell. & - & 0.50 & - & 0.61 & - & 0.07 & - & 1.18 & Ane & $\mathrm{D}$ \\
\hline Guarea guidonia (L.) Sleumer & 1.75 & 2.75 & 1.54 & 2.74 & 1.45 & 1.23 & 4.75 & 6.71 & Zoo & $\mathrm{E}$ \\
\hline Trichilia elegans A. Juss. & 0.75 & 2.00 & 0.62 & 2.13 & 0.18 & 0.75 & 1.55 & 4.87 & Zoo & $\mathrm{E}$ \\
\hline Trichilia palida Sw. & - & 0.50 & - & 0.61 & - & 0.20 & - & 1.30 & Zoo & $\mathrm{E}$ \\
\hline \multicolumn{11}{|l|}{ Monimiaceae } \\
\hline Mollinedia widgrenii A. DC. & 1.00 & - & 0.62 & - & 0.62 & - & 2.24 & - & Zoo & $\mathrm{E}$ \\
\hline \multicolumn{11}{|l|}{ Moraceae } \\
\hline Ficus citrifolia Mill. & 0.25 & - & 0.31 & - & 0.17 & - & 0.73 & - & Zoo & $\mathrm{E}$ \\
\hline Ficus trigona L. f. & 0.25 & 0.50 & 0.31 & 0.61 & 0.40 & 0.70 & 0.96 & 1.81 & Zoo & $\mathrm{E}$ \\
\hline Maclura tinctoria (L.) Steud & - & 0.25 & - & 0.30 & - & 0.05 & - & 0.60 & Zoo & $\mathrm{D}$ \\
\hline \multicolumn{11}{|l|}{ Myrtaceae } \\
\hline Campomanesia velutina (Cambess.) O. Berg & 1.00 & 1.00 & 1.23 & 1.22 & 0.32 & 0.43 & 2.55 & 2.65 & Zoo & $\mathrm{D}$ \\
\hline Eugenia florida DC. & 0.50 & 0.50 & 0.62 & 0.61 & 0.23 & 0.13 & 1.35 & 1.24 & Zoo & $\mathrm{E}$ \\
\hline Myrcia splendens (Sw.) DC. & 1.75 & 0.25 & 2.16 & 0.30 & 0.48 & 0.29 & 4.39 & 0.84 & Zoo & $\mathrm{E}$ \\
\hline Myrcia tomentosa (Aubl.) DC. & 0.75 & - & 0.93 & - & 0.23 & - & 1.90 & - & Zoo & $\mathrm{D}$ \\
\hline Psidium guajava L. & 0.25 & 0.25 & 0.31 & 0.30 & 0.04 & 0.04 & 0.60 & 0.59 & Zoo & $\mathrm{E}$ \\
\hline
\end{tabular}

$\mathrm{RD}=$ relative density; $\mathrm{RF}=$ relative frequency; RBA = relative basal area; IV = importance value; $\mathrm{DS}=$ dispersal syndrome; Dec $=$ deciduousness; Ane $=$ anemochoric species; Auto = autochoric species; Zoo = zoochoric species; $\mathrm{D}=$ deciduous; $\mathrm{E}=$ evergreen. $* \mathrm{DBH} \geq 5 \mathrm{~cm} . * *$ Distant from the reservoir margin. $* * *$ Close to the reservoir margin. 
Table 3. Continued...

\begin{tabular}{|c|c|c|c|c|c|c|c|c|c|c|}
\hline \multirow{2}{*}{ Family/species } & \multicolumn{2}{|c|}{ RD } & \multicolumn{2}{|c|}{ RF } & \multicolumn{2}{|c|}{ RBA } & \multicolumn{2}{|c|}{ IV (\%) } & \multirow{2}{*}{ DS } & \multirow{2}{*}{ Dec } \\
\hline & $\mathbf{A}$ & B & $\mathbf{A}$ & $\mathbf{B}$ & $\mathbf{A}$ & B & $\mathbf{A}$ & $\mathbf{B}$ & & \\
\hline \multicolumn{11}{|l|}{ Nyctaginaceae } \\
\hline Guapira venosa (Choisy) Lundell & 0.25 & - & 0.31 & - & 0.16 & - & 0.72 & - & Zoo & $\mathrm{E}$ \\
\hline \multicolumn{11}{|l|}{ Piperaceae } \\
\hline Piper aduncum L. & - & 7.00 & - & 6.38 & - & 3.09 & - & 16.47 & Zoo & $\mathrm{E}$ \\
\hline Piper amalago L. & 2.00 & - & 1.85 & - & 0.54 & - & 4.39 & - & Zoo & $\mathrm{E}$ \\
\hline \multicolumn{11}{|l|}{ Primulaceae } \\
\hline Ardisia ambigua $\mathrm{Mez}$ & - & 0.75 & - & 0.91 & - & 0.20 & - & 1.86 & Zoo & $\mathrm{E}$ \\
\hline Myrsine umbellata Mart & 4.75 & 20.75 & 5.25 & 14.59 & 4.10 & 16.51 & 14.10 & 51.85 & Zoo & $\mathrm{E}$ \\
\hline \multicolumn{11}{|l|}{ Rhamnaceae } \\
\hline Rhamnidium elaeocarpum Reissek & 8.25 & 6.25 & 8.02 & 5.47 & 3.63 & 10.25 & 19.90 & 21.97 & Zoo & $\mathrm{D}$ \\
\hline \multicolumn{11}{|l|}{ Rubiaceae } \\
\hline Alibertia edulis (L. C. Rich.) A. Rich. ex DC. & 0.25 & - & 0.31 & - & 0.06 & - & 0.62 & - & Zoo & $\mathrm{E}$ \\
\hline Chomelia pohliana Müll.Arg & 0.25 & 1.50 & 0.31 & 1.82 & 0.04 & 0.62 & 0.60 & 3.94 & Zoo & $\mathrm{E}$ \\
\hline Coutarea hexandra (Jacq.) K. Schum. & 1.00 & 0.75 & 1.23 & 0.61 & 0.43 & 0.24 & 2.66 & 1.59 & Ane & $\mathrm{D}$ \\
\hline Simira sampaioana (Standl.) Steyerm. & 0.25 & - & 0.31 & - & 0.12 & - & 0.68 & - & Ane & $\mathrm{D}$ \\
\hline \multicolumn{11}{|l|}{ Rutaceae } \\
\hline Zanthoxylum riedelianum Engl. & 0.50 & - & 0.31 & - & 0.13 & - & 0.94 & - & Zoo & $\mathrm{E}$ \\
\hline \multicolumn{11}{|l|}{ Salicaceae } \\
\hline Casearia gossypiosperma Briq & 1.75 & 0.25 & 1.85 & 0.30 & 0.43 & 0.03 & 4.03 & 0.59 & Zoo & $\mathrm{D}$ \\
\hline Casearia rupestris Eichler & 1.75 & - & 1.54 & - & 1.27 & - & 4.56 & - & Zoo & $\mathrm{D}$ \\
\hline Casearia sylvestris $\mathrm{Sw}$. & 0.25 & - & 0.31 & - & 0.03 & - & 0.59 & - & Zoo & $\mathrm{E}$ \\
\hline \multicolumn{11}{|l|}{ Sapindaceae } \\
\hline Allophylus racemosus Sw. & 1.50 & 0.25 & 1.54 & 0.30 & 0.40 & 0.07 & 3.44 & 0.62 & Zoo & $\mathrm{E}$ \\
\hline Cupania vernalis Cambess. & - & 0.25 & - & 0.30 & - & 0.03 & - & 0.59 & Zoo & $\mathrm{E}$ \\
\hline Dilodendron bipinnatum Radlk & 3.50 & 1.25 & 3.09 & 1.52 & 2.12 & 0.56 & 8.71 & 3.33 & Zoo & $\mathrm{D}$ \\
\hline Matayba elaeagnoides Radlk. & 0.25 & 0.50 & 0.31 & 0.61 & 0.08 & 0.10 & 0.64 & 1.21 & Zoo & $\mathrm{E}$ \\
\hline \multicolumn{11}{|l|}{ Sapotaceae } \\
\hline $\begin{array}{l}\text { Chrysophyllum gonocarpum (Mart. \& } \\
\text { Eichler) Engl. }\end{array}$ & 0.50 & - & 0.62 & - & 0.39 & - & 1.50 & - & Zoo & $\mathrm{E}$ \\
\hline Pouteria gardneri (Mart. \& Miq.) Baehni & 0.25 & 0.50 & 0.31 & 0.61 & 0.63 & 0.14 & 1.19 & 1.25 & Zoo & $\mathrm{E}$ \\
\hline \multicolumn{11}{|l|}{ Urticaceae } \\
\hline Cecropia pachystachya Trécul & 0.50 & 2.25 & 0.62 & 2.74 & 0.44 & 2.46 & 1.56 & 7.44 & Zoo & $\mathrm{E}$ \\
\hline Urera baccifera (L.) Gaudich. ex Wedd. & 1.00 & - & 0.93 & - & 0.75 & - & 2.67 & - & Zoo & $\mathrm{D}$ \\
\hline
\end{tabular}

$\mathrm{RD}=$ relative density; $\mathrm{RF}=$ relative frequency; $\mathrm{RBA}=$ relative basal area; $\mathrm{IV}=$ importance value; $\mathrm{DS}=$ dispersal syndrome; Dec $=$ deciduousness; Ane $=$ anemochoric species; Auto $=$ autochoric species; Zoo = zoochoric species; $\mathrm{D}=$ deciduous; $\mathrm{E}=$ evergreen. $* \mathrm{DBH} \geq 5 \mathrm{~cm}$. **Distant from the reservoir margin. $* *$ Close to the reservoir margin.

exclusive to sector B (Table 3). The five species with the highest values in the importance value (IV) in sector A were Myracrodruon urundeuva (18\%), Rhamnidium elaeocarpum (7\%), Guazuma ulmifolia (6\%), Cariniana estrellensis (6\%), and Deguelia costata (6\%), representing $43 \%$ of total IV (Table 3 ). In sector B, the five species with the highest values in the IV were Myrsine umbellata (17\%), Myracrodruon urundeuva (12\%), Rhamnidium elaeocarpum (7\%), Anadenanthera colubrina (6\%), and Piper aduncum (5\%), representing $47 \%$ of total IV (Table 3 ).

Although most species were common to both forest sectors, the relative density of some species significantly differ with regard to disturbance level (Table 3 ). The species Myracrodruon urundeuva $(\mathrm{U}=15.5, \mathrm{p}<0.01)$ and
Cariniana estrellensis $(\mathrm{U}=25.0, \mathrm{p}<0.05)$ showed a significantly higher density in sector A. In turn, higher densities of Myrsine umbellate $(\mathrm{U}=15.0, \mathrm{p}<0.01)$, Piper aduncum $(\mathrm{U}=10.0, \mathrm{p}<0.01)$, Luehea divaricata $(\mathrm{U}=29.0$, $\mathrm{p}<0.05)$, and Cecropia pachystachya $(\mathrm{U}=27.0, \mathrm{p}<0.05)$ were observed in sector B.

The cluster based only in the presence/absence did not results in floristic groups with similarity $>50 \%$, suggesting a high floristic heterogeneity (Figure 1a). Otherwise, the cluster based on the number of individuals results in a group with similarity $>50 \%$, consisting of areas $1,2,3,4$, 7,8 and A (far from the reservoir) (see Figure 1b), mainly due to the high number of individuals of the trees species Anadenanthera colubrina, Myracrodruon urundeuva, 

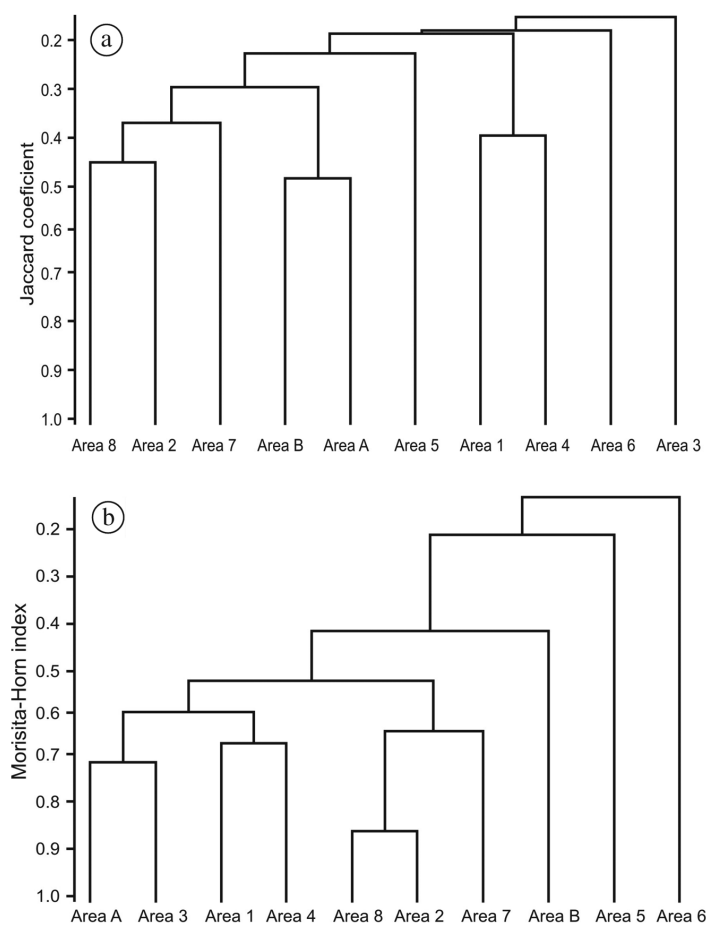

Figure 1. Similarity cluster based on presence/absence of species (a) and species abundance (b) of nine deciduous dry forests in the Southeast Brazilian region. (a) Sector distant from the reservoir margin. (b) Sector close to the reservoir margin.

Guazulma ulmifolia, and Piptadenia gonoacantha - all of them frequently observed in dry forests. This first group could be subdivided into two groups, one consisting of areas 1, 3, 4 and A, and the other subgroup consisting of areas 2, 7 and 8. The first group had species such as Aralia warmingii and many species of the genus Trichilia, while the second had a high abundance of Campomanesia velutina, Casearia rupestris, Myracrodruon urundeuva, and Tabebuia roseoalba. However, sector B, close to the reservoir margin, did not form a group with similarity $>50 \%$ in this cluster based on the number of individuals, perhaps due the occurrence of species adapted to soils with a higher water supply, such as Myrsine umbellata, Inga sessilis, Guarea guidonia, Piper aduncum, and Cecropia pachystachya.

\section{Discussion}

The differences on the floristic parameters of sector A and sector B may be a consequence of the dam. The lower floristic diversity of sector B may reflect a stressful environmental condition, as low species diversity happen in disturbed environments (Connell, 1971). Water level elevation due to the dam changes the ground moisture, constituting an environmental filter; the role of an environmental filter is selecting species with similar traits able to survive and reproduce in a certain place (Sobral and Cianciaruso, 2012). In this study, the species less able to adapt to the new conditions (high soil moisture) showed changes in their density and, in the worst scenario, they may be locally extinct (Chase, 2003). Soil influenced by the dam became saturated with water and the dry forest turned into a riparian-like forest, thus, changes in flora are expected (Gusson et al., 2011).

The influence of the number of trees and the basal area was much higher close to the dam than distant to it; this was also higher when compared to non-impacted dry forests whose results, presented in other studies, were compared to the results from this paper (Table 1). These events are probably due to the combination of two abiotic factors, higher soil moisture because of closeness to a water body and good soil nutrition, which constitutes a natural feature of these forests (Gusson et al., 2011). Drought reduces plant growth (Churkina and Running, 1998; Hinckley et al., 1979), since there is a decreased plant photosynthetic capacity (Martin et al., 1994); otherwise, soil fertility increases the plant net yield (Malhi et al., 2004; Phillips et al., 2004). Therefore, combining these factors may increase plant growth, facilitating the establishment of many trees and an increased basal area.

The higher percentage of zoochoric species in sector B when compared to sector A confirms the importance of maintaining the biotic dispersal agents in the forest, mainly in humid patches. Dispersal by animals is more common in tropical humid forests than in seasonal forests (Howe and Smallwood, 1982; Toniato and Oliveira-Filho, 2004). Therefore, the influence of the dam after 15 years showed to be able to change the predominant dispersal mechanism in patches close to the reservoir margin.

Anemochoric dispersal is favored in dry environments (Lima et al., 2010), because the dry diaspores can be easily carried, however, high moisture may wet the seeds, increase their weight, and make them more difficult to be carried (Talora and Morellato, 2000; Spina et al., 2001). Leaf deciduousness also facilitates wind dispersal, because less foliage means less barriers to wind (Spina et al., 2001). Therefore, many anemochoric species lose all their leaves in winter, such as Myracrodruon urundeuva, Piptadenia gonoacantha, and Anadenanthera colubrina, three important species found in sector A. This way, a long dry period without rainfall reduce soil moisture and it may be regarded as a significant stress factor to plants, mainly due to the evapotranspiration process; otherwise, it increases wind dispersal in deciduous plants. Anemochoric plants also can reach a high height by maximizing dispersal effectiveness, due to increased exposition to wind (Pires-O'Brien and O'Brien, 1995; Nunes et al., 2003), thus, many important species in sector A are deciduous, anemochoric, and form a canopy (except Deguelia costata)

However, this dispersal mechanism has disadvantages in patches close to the dam. First, because a higher water supply favors the establishment of species non-adapted to dry environments, i.e. less deciduous and more evergreen species will occur, creating barriers to dispersal (Fathi-Moghadam, 2007; Cassiani et al., 2008). Second, because water can be used by zoochoric species to produce bigger and more succulent fruits (Pinheiro and Ribeiro, 2001; 
Rodrigues et al., 2010), increasing their presence in the fauna and, as a consequence, increasing the dispersal of their seeds (Gusson et al., 2011). A clear example was Myrsine umbellata, a zoochoric and evergreen species with low tolerance to water deficit (Silva and Dillenburg, 2007), but it dominates sector B after 15 years under the influence of the dam. Dry forest associated to mountains are usually very fertile due to the presence of basaltic rocks and there was no other environmental filter able to hinder the establishment of species typical of dry forests; then, the community close to the dam changed its physiognomy after 15 years. Previously, it was a deciduous dry forest with leaves falling in winter and predominant anemochory; now, it is a semideciduous forest with many zoochoric species. This increases the community heterogeneity, because different species are included into the community and this may be regarded as a strong impact on these forests.

The sampled species with high density in both sectors were commonly found in other forests within the same geographical region (Santos et al., 2007; Siqueira et al., 2009), except Myrsine umbellata. Most of them are distributed into the Brazilian dry forests, and the most frequently observed species are Myracrodruon urundeuva and Anadenanthera colubrina (Prado and Gibbs, 1993; Araújo et al., 1997; Linares-Palomino et al., 2009). Meanwhile, in all selected studies, M. umbellata had a limited importance, then, it is suggested that the high density of this species only in sector B is due to the dam influence. Perhaps, changes to soil moisture due to the dam favor the establishment of this species. Other study addressing M. umbellata on soils with high and low moisture concluded that this species has a low tolerance to hydric deficit, needing a soil with continued access to water in order to establish in warm location with high solar irradiance (Silva and Dillenburg, 2007).

The results make evident a high floristic similarity between sector A, distant from water, and other deciduous forests within the same geographical region. Sector B shows a higher richness of species adapted to high moisture on the soil, such as Myrsine umbellata, Inga sessilis, Guarea guidonia, Piper aduncum, and Cecropia pachystachya; the latter also indicated high moisture soils, water bodies, or superficial groundwater (Batista et al., 2008). Guarea guidonia and Inga sessilis are typical of riparian forest associated to rivers and alluvial plains and valleys, i.e. humid environments (Lorenzi, 1998).

The species showing the most significant values in sector A was Myracrodruon urundeuva, due to its thick individuals established before the dam was built. Meanwhile, few young individuals of this species were found close to the lakeshore, and this fact suggests that M. urundeuva has some problem to establish close to the reservoir. Other studies showed a high mortality and a low prevalence of seedlings, saplings, and young individuals of this species under high soil moisture conditions after the dam was built (Gusson et al., 2011).

The presence/absence did not formed coherent groups and it is possible to point out that there is a high heterogeneity in this forest. Otherwise, the structural similarity analysis divided it into sectors $\mathrm{A}$ and $\mathrm{B}$; the first maintains a high number of species typical of dry forests and the second, after 15 years under the influence of the dam, had many species adapted to a high soil moisture condition. Thus, the regional pool of species did not change a lot, but the forest structure became very different: now, the dry forest under study is clearly more heterogeneous, with some species adapted to water saturation. Several of these species have traits commonly found in humid forests, such as zoochoric dispersal and evergreen leaves, then, it is possible to conclude that 15 years undergoing the impacts of a dam were enough to cause several changes to the forest structure, with consequences to vegetations structure, confirming the hypothesis assumed in this paper and also, is probably that the number off zoochory species will, in long-term, influence the associated fauna.

\section{Acknowledgements}

The authors thank to the Foundation for Research Support of the State of Minas Gerais (FAPEMIG) and the Coordination for the Improvement of Higher Level Personnel (CAPES), for the financial support (Process 2,498/09-0).

\section{References}

ACKER, SA., GREGORY, S., LIENKAEMPER, G., MCKEE, WA., SWANSON, FJ. and MILLER, SD., 2003. Composition, complexity, and tree mortality in riparian forests in the central Western Cascades of Oregon. Forest Ecology and Management, vol. 173, no. 1-3, p. 293-308. http://dx.doi.org/10.1016/S03781127(02)00005-1.

ALVES, KA. and ROSA, R., 2008. Espacialização de dados climáticos do cerrado mineiro. Revista Horizonte Científico, vol. 8, p. 1-28.

ARAÚJO, GM., RODRIGUES, LA. and IVIZI, L., 1997. Estrutura fitossociológica e fenologia de espécies lenhosas de mata decídua em Uberlândia, MG. In LEITE, LL. and SAITO, CH. (Eds.). Contribuição ao conhecimento ecológico do cerrado. Brasília: Editora Universidade de Brasília. p. 22-28.

BATISTA, CUN., MEDRI, ME., BIANCHINI, E., MEDRI, C. and PIMENTA, JA., 2008. Tolerância à inundação de Cecropia pachystachya Trec. (Cecropiaceae): aspectos ecofisiológicos e morfoanatômicos. Acta Botanica Brasílica, vol. 22, no. 1, p. 91-98. http://dx.doi.org/10.1590/S0102-33062008000100012.

BERTRAND, G., GOLDSCHEIDER, N., GOBAT, JM. and HUNKELER, D., 2012. Review: from multi-scale conceptualization to a classification system for inland groundwater-dependent ecosystems. Hydrogeology Journal, vol. 10, p. 1-21.

CARVALHO, DA., OLIVEIRA FILHO, AT. and VILELA, EA., 1999. Florística e fitossociologia da vegetação arbóreo-arbustiva de floresta ripária decidua do baixo Paranaíba (Santa Vitória, Minas Gerais). Revista Árvore, vol. 23, p. 311-320.

CASSIANI, M., KATUL, GG. and ALBERTSON, JD., 2008. The effects of canopy leaf area index on airflow across forest edges: Large-eddy simulation and analytical results. BoundaryLayer Meteorology, vol. 126, no. 3, p. 433-460. http://dx.doi. org/10.1007/s10546-007-9242-1. 
CHASE, JM., 2003. Community assembly: when should history matter? Oecologia, vol. 136, no. 4, p. 489-498. http://dx.doi. org/10.1007/s00442-003-1311-7. PMid:12836009.

CHASE, MW. and REVEAL, JL., 2009. A phylogenetic classification of the land plants to accompany APG III. Botanical Journal of the Linnean Society, vol. 161, no. 2, p. 122-127. http://dx.doi. org/10.1111/j.1095-8339.2009.01002.x.

CHURKINA, G. and RUNNING, SW., 1998. Contrasting climatic controls on the estimated productivity of global terrestrial biomes. Ecosystems (New York, N.Y.), vol. 1, no. 2, p. 206-215. http:// dx.doi.org/10.1007/s100219900016.

CONNELL, JH., 1971. On the role of natural enemies in preventing competitive exclusion in some marine animals and in forest trees. In DEN BOER, PJ. and GRADWELL, GR. (Eds.). Dynamics of populations. Wageningen: Centre for Agricultural Publishing and Documentation. p. 298-312.

COTTAM, G. and CURTIS, JT., 1956. The use of distance measures in phytosociological sampling. Ecology, vol. 37, no. 3, p. 451-460. http://dx.doi.org/10.2307/1930167.

EVANS, A., STREZOV, V. and EVANS, TJ., 2009. Assessment of sustainability indicators for renewable energy technologies. Renewable \& Sustainable Energy Reviews, vol. 13, no. 5, p. 10821088. http://dx.doi.org/10.1016/j.rser.2008.03.008.

FAGUNDES, LM., CARVALHO, DAD., VAN DEN BERG, E., MELO MARQUES, JJGS. and MACHADO, ELM., 2007. Florística e estrutura do estrato arbóreo de dois fragmentos de florestas decíduas às margens do rio Grande, em Alpinópolis e Passos, MG, Brasil. Acta Botanica Brasilica, vol. 21, p. 65-78. http://dx.doi.org/10.1590/S0102-33062007000100007.

FATHI-MOGHADAM, M., 2007. Characteristics and mechanics of tall vegetation for resistance to flow. African Journal of Biotechnology, vol. 6, p. 475-480.

GILES, J., 2006. Methane quashes green credentials of hydropower. Nature, vol. 444, no. 7119, p. 524-525. http://dx.doi. org/10.1038/444524b. PMid:17136056.

GUNDERSEN, P., LAURÉN, A., FINÉR, L., RING, E., KOIVUSALO, H., SAETERSDAL, M., WESLIEN, JO., SIGURDSSON, BD., HÖGBOM, L., LAINE, J. and HANSEN, K., 2010. Environmental services provided from riparian forests in the Nordic countries. Ambio, vol. 39, no. 8, p. 555-566. http:// dx.doi.org/10.1007/s13280-010-0073-9. PMid:21141775.

GUO, Z., LI, Y., XIAO, X., ZHANG, L. and GAN, Y., 2007. Hydroelectricity production and forest conservation in watersheds. Ecological Applications: a Publication of the Ecological Society of America, vol. 17, no. 6, p. 1557-1562. http://dx.doi.org/10.1890/060840.1. PMid:17913122.

GUSSON, AE., VALE, VS., OLIVEIRA, AP., LOPES, SF., DIAS-NETO, OC., ARAÚJO, GM. and SCHIAVINI, I., 2011. Interferência do aumento de umidade do solo nas populações de Myracrodruon urundeuva Allemão e Anadenanthera colubrina (Vell.) Brenan em reservatórios artificiais de Usinas Hidrelétricas. Scientia Florestalis, vol. 39, p. 35-41.

HINCKLEY, TM., DOUGHERTY, PM., LASSOIE, JP., ROBERTS, JE. and TESKEY, RO., 1979. Severe drought - Impact on tree growth, phenology, net photosynthetic rate and water relations. American Midland Naturalist, vol. 102, no. 2, p. 307-316. http:// dx.doi.org/10.2307/2424658.

HOWE, HF. and SMALLWOOD, J., 1982. Ecology of seed dispersal. Annual Review of Ecology and Systematics, vol. 13, no. 1, p. 201-228. http://dx.doi.org/10.1146/annurev.es.13.110182.001221.
JANSSON, R., NILSSON, C., DYNESIUS, M. and ANDERSSON, E., 2000. Effects of river regulation on river-margin vegetation: a comparison of eight boreal rivers. Ecological Applications, vol. 10 , no. 1 , p. 203-224. http://dx.doi.org/10.1890/10510761(2000)010[0203:EORROR]2.0.CO;2.

LIMA, MS., DAMASCENO-JÚNIOR, GA. and TANAKA, MO., 2010. Aspectos estruturais da comunidade arbórea em remanescentes de floresta estacional decidual, em Corumbá, MS, Brasil. Revista Brasileira de Botanica. Brazilian Journal of Botany, vol. 33, no. 3, p. 437-453. http://dx.doi.org/10.1590/ S0100-84042010000300007.

LINARES-PALOMINO, R., OLIVEIRA-FILHO, AT. and PENNINGTON, RT., 2009. Neotropical Seasonally dry forests: diversity, endemism, and biogeography of woody plants. In DIRZO, R., YOUNG, HS., MOONEY, HA. and CEBALLOS, G. (Eds.). Seasonally dry tropical forests: ecology and conservation. Washington, DC: Island Press. 407 p.

LOPES, SF., SCHIAVINI, I., OLIVEIRA, A. and VALE, VS., 2012. An ecological comparison of floristic composition in Seasonal Semideciduous Forest in southeast Brazil: implications for conservation. International Journal of Forestry Research, vol. 2012, p. 1-14. http://dx.doi.org/10.1155/2012/537269.

LOREAU, M., NAEEM, S., INCHAUSTI, P., BENGTSSON, J., GRIME, JP., HECTOR, A., HOOPER, DU., HUSTON, MA., RAFFAELLI, D., SCHMID, B., TILMAN, D. and WARDLE, DA., 2001. Biodiversity and ecosystem functioning: current knowledge and future challenges. Science, vol. 294, no. 5543, p. 804-808. http://dx.doi.org/10.1126/science.1064088. PMid:11679658.

LORENZI, H., 1998. Árvores brasileiras: manual de identificação e cultivo de plantas arbóreas nativas do Brasil. 2nd ed. Nova Odessa: Editora Plantarum.

MAGURRAN, AE., 2004. Measuring biological diversity. Malden: Blackwell Science. 256 p.

MALHI, Y., BAKER, TR., PHILLIPS, OL., ALMEIDA, S., ALVAREZ, E., ARROYO, L., CHAVE, J., CZIMCZIK, CI., DI-FIORE, A., HIGUCHI, N., KILLEEN, TJ., LAURANCE, SG., LAURANCE, WF., LEWIS, SL., MONTOYA, LMM., MONTEAGUDO, A., NEILL, DA., VARGAS, PN., PATINO, S., PITMAN, NCA., QUESADA, CA., SALOMAO, R., SILVA, JNM., LEZAMA, AT., MARTINEZ, RV., TERBORGH, J., VINCETI, B. and LLOYD, J., 2004. The above-ground coarse wood productivity of 104 Neotropical forest plots. Global Change Biology, vol. 10, no. 5, p. 563-591. http://dx.doi.org/10.1111/j.15298817.2003.00778.x.

MALLIK, AU. and RICHARDSON, JS., 2009. Riparian vegetation change in upstream and downstream reaches of three temperate rivers dammed for hydroelectric generation in British Columbia, Canada. Ecological Engineering, vol. 35, no. 5, p. 810-819. http:// dx.doi.org/10.1016/j.ecoleng.2008.12.005.

MARTIN, CE., LOESCHEN, VS. and BORCHERT, R., 1994. Photosynthesis and leaf longevity in trees of a tropical deciduous forest in Costa-Rica. Photosynthetica, vol. 30, p. 341-351.

MILES, L., NEWTON, AC., DEFRIES, RS., RAVILIOUS, C., MAY, I., BLYTH, S., KAPOS, V. and GORDON, JE., 2006. A global overview of the conservation status of tropical dry forests. Journal of Biogeography, vol. 33, no. 3, p. 491-505. http://dx.doi. org/10.1111/j.1365-2699.2005.01424.x.

MURPHY, PG. and LUGO, AE., 1986. Ecology of Tropical Dry Forest. Annual Review of Ecology and Systematics, vol. 17, no. 1, p. 67-88. http://dx.doi.org/10.1146/annurev.es.17.110186.000435. 
NILSSON, C. and BERGGREN, K., 2000. Alterations of riparian ecosystems caused by river regulation. Bioscience, vol. 50, no. 9, p. 783-792. http://dx.doi.org/10.1641/0006-3568(2000)050[0783:AO RECB]2.0.CO;2.

NILSSON, C. and SVEDMARK, M., 2002. Basic principles and ecological consequences of changing water regimes: riparian plant communities. Environmental Management, vol. 30, no. 4, p. 468-480. http://dx.doi.org/10.1007/s00267-002-2735-2. PMid:12481914

NILSSON, C., JANSSON, R. and ZINKO, U., 1997. Long-term responses of river-margin vegetation to water-level regulation. Science, vol. 276, no. 5313, p. 798-800. http://dx.doi.org/10.1126/ science.276.5313.798. PMid:9115205.

NILSSON, C., REIDY, CA., DYNESIUS, M. and REVENGA, C., 2005. Fragmentation and flow regulation of the world's large river systems. Science, vol. 308, no. 5720, p. 405-408. http:// dx.doi.org/10.1126/science.1107887. PMid:15831757.

NUNES, YR., MENDONÇA, AVR., BOTEZELLI, L., MACHADO, ELM. and OLIVEIRA-FILHO, AT., 2003. Variações da fisionomia, diversidade e composição de guildas da comunidade arbórea em um fragmento de floresta semidecidual em Lavras, MG. Acta Botanica Brasílica, vol. 17, no. 2, p. 213-229. http://dx.doi. org/10.1590/S0102-33062003000200005.

PALMER, GC. and BENNETT, AF., 2006. Riparian zones provide for distinct bird assemblages in forest mosaics of south-east Australia. Biological Conservation, vol. 130, no. 3, p. 447-457. http://dx.doi.org/10.1016/j.biocon.2006.01.006.

PHILLIPS, OL., BAKER, TR., ARROYO, L., HIGUCHI, N., KILLEEN, TJ., LAURANCE, WF., LEWIS, SL., LLOYD, J., MALHI, Y., MONTEAGUDO, A., NEILL, DA., VARGAS, PN., SILVA, JNM., TERBORGH, J., MARTÍNEZ, RV., ALEXIADES, M., ALMEIDA, S., BROWN, S., CHAVE, J., COMISKEY, JA., CZIMCZIK, CI., DI FIORE, A., ERWIN, T., KUEBLER, C., LAURANCE, SG., NASCIMENTO, HEM., OLIVIER, J., PALACIOS, W., PATIÑO, S., PITMAN, NCA., QUESADA, CA., SALDIAS, M., LEZAMA, AT. and VINCETI, B., 2004. Pattern and process in Amazon tree turnover, 1976-2001. Philosophical Transactions of the Royal Society of London. Series B, Biological Sciences, vol. 359, no. 1443, p. 381-407. http://dx.doi.org/10.1098/ rstb.2003.1438. PMid:15212092.

PINHEIRO, F. and RIBEIRO, JF., 2001. Síndromes de dispersão de sementes em Matas de Galeria do Distrito Federal. In RIBEIRO, JF., FONSECA, CE. and SILVA, JC. (Eds.). Cerrado: caracterização e recuperação de matas de galeria. Planaltina: Embrapa. p. 335-375.

PIRES-O'BRIEN, MJ. and O'BRIEN, CM., 1995. Ecologia e modelamento de florestas tropicais. Belém: FCAP.

PRADO, DE. and GIBBS, PE., 1993. Patterns of species distributions in the dry seasonal forest South America. Annals of the Missouri Botanical Garden, vol. 80, no. 4, p. 902-927. http:// dx.doi.org/10.2307/2399937.

RODRIGUES, VHP., LOPES, SF., ARAÚJO, GM. and SCHIAVINI, I., 2010. Composição, estrutura e aspectos ecológicos da floresta ciliar do rio Araguari no Triângulo Mineiro. Hoehnea, vol. 37, no. 1, p. 87-105. http://dx.doi.org/10.1590/S2236-89062010000100006.

SANTOS, RM., VIEIRA, FA, GUSMÃO, E. and NUNES, YRF., 2007. Florística e estrutura de uma floresta estacional decidual no Parque Municipal da Sapucaia, Montes Claros, MG. Cerne, vol. 13, p. $248-256$.
SHEPHERD, GJ., 2004. Fitopac-Shell. Campinas: Universidade Estadual de Campinas. Version 1.6.4.

SIDLE, RC., ZIEGLER, AD., NEGISHI, JN., NIK, AR., SIEW, R. and TURKELBOOM, F., 2006. Erosion processes in steep terrain - truths, myths, and uncertainties related to forest management in Southeast Asia. Forest Ecology and Management, vol. 224, no. 1-2, p. 199-225. http://dx.doi.org/10.1016/j.foreco.2005.12.019.

SILVA, LGR. and DILLENBURG, LR., 2007. Water relations of tree species growing on a rock outcrop in the "Parque Estadual de Itapuã", RS. Revista Brasileira de Botanica. Brazilian Journal of Botany, vol. 30, no. 4, p. 703-711. http://dx.doi.org/10.1590/ S0100-84042007000400015.

SIQUEIRA, AS., ARAÚJO, GM. and SCHIAVINI, I., 2009. Estrutura do componente arbóreo e características edáficas de dois fragmentos de floresta estacional decidual no vale do rio Araguari, MG, Brasil. Acta Botanica Brasílica, vol. 23, no. 1, p. 10-21. http://dx.doi.org/10.1590/S0102-33062009000100003.

SOBRAL, FL. and CIANCIARUSO, MV., 2012. Estrutura filogenética e funcional de assembléias: (re) montando a ecologia de comunidades em diferentes escalas espaciais. Bioscience Journal, vol. 28, p. 617-631.

SOUZA, JP., ARAÚJO, GM. and HARIDASAN, M., 2007. Influence of soil fertility on the distribution of tree species in a deciduous forest in the Triângulo Mineiro region of Brazil. Plant Ecology, vol. 191, no. 2, p. 253-263. http://dx.doi.org/10.1007/ s11258-006-9240-2.

SPINA, AP., FERREIRA, WM. and LEITÃO-FILHO, HF., 2001. Floração, frutificação e síndromes de dispersão de uma comunidade de floresta de brejo na região de Campinas (SP). Acta Botanica Brasílica, vol. 15, no. 3, p. 349-368. http://dx.doi.org/10.1590/ S0102-33062001000300006.

TALORA, DC. and MORELLATO, PC., 2000. Fenologia de espécies arbóreas em floresta de planície litorânea do sudeste do Brasil. Revista Brasileira de Botanica. Brazilian Journal of Botany, vol. 23, no. 1, p. 13-26. http://dx.doi.org/10.1590/ S0100-84042000000100002.

TONIATO, MTZ. and OLIVEIRA-FILHO, AT., 2004. Variations in tree community composition and structure in a fragment of tropical semideciduous forest in southeastern Brazil related to different human disturbance histories. Forest Ecology and Management, vol. 198, no. 1-3, p. 319-339. http://dx.doi. org/10.1016/j.foreco.2004.05.029.

TRUFFER, B., BRATRICH, C., MARKARD, J., PETER, A., WUEST, A. and WEHRLI, B., 2003. Green Hydropower: The contribution of aquatic science research to the promotion of sustainable electricity. Aquatic Sciences, vol. 65, p. 99-110.

VALE, VS., SCHIAVINI, I., OLIVEIRA, AP. and GUSSON, AE., 2010. When ecological functions are more important than richness: A conservation approach. Journal of Ecology and the Natural Environment, vol. 2, p. 270-280.

VAN DER PIJL, L., 1982. Principles of dispersal in higher plants. 5th ed. Berlin: Springer Verlag.

WERNECK, MS., FRANCESCHINELLI, EV. and TAMEIRÃONETO, E., 2000. Mudanças na florística e estrutura de uma floresta decidua durante um período de quarto anos (1994-1998), na região do Triângulo Mineiro, MG. Revista Brasileira de Botânica, vol. 23, no. 4, p. 401-413. http://dx.doi.org/10.1590/ S0100-84042000000400006. 